\title{
PATRIMONIO PALEONTOLÓGICO (INVERTEBRADOS) DE LAS ISLAS CANARIAS EN EL MUSEO NACIONAL DE CIENCIAS NATURALES-CSIC
}

\author{
Ángel MONTERO', Carmen DIÉGUEZ' \\ y María Ángeles RAMOS \\ ' Jardín Botánico de Córdoba. Avda. Linneo s/n. 14004 Córdoba \\ ? Museo Nacional de Ciencias Naturales. c/ José Gutiérrez Abascal nº 2. 28006 Madrid
}

\begin{abstract}
Montero, Á., Diéguez, C. y Ramos, M.Á. 2001. Patrimonio paleontológico (invertebrados) de las Islas Canarias en el Museo Nacional de Ciencias Naturales-CSIC. [Palaeontological heritage (invertebrates) from the Canary Islands in the Museo Nacional de Ciencias Naturales-CSIC.] Revista Española de Paleontología, no extraordinario, 81-84. ISSN 0213-6937.
\end{abstract}

\begin{abstract}
Material from the Canary Islands (molluscs and ichnofossils) is housed in the collection of invertebrate palaeontology in the Museo Nacional de Ciencias Naturales-CSIC, Madrid. Apart from a single donation these correspond to collecting trips in the late 1800 s and early 1900s carried out by a few people, although the most important acquisitions are due to E. Hernández Pacheco as a result of his geological investigation of the islands, which was commisioned by the Real Sociedad Española de Historia Natural. The entire collection has been revised taxonomically and with regard to the documentation of its origins. This has shown its undeniable historical interest.
\end{abstract}

Keywords: Paleontological heritage, fossil invertebrates, Canary Islands, Spain, molluscs, ichnofossils.

\section{RESUMEN}

Entre los fondos paleontológicos custodiados en el Museo Nacional de Ciencias Naturales-CSIC, se encuentran ejemplares de invertebrados fósiles procedentes de las Islas Canarias. Este patrimonio paleontológico, consistente en ejemplares atribuibles a diferentes especies de moluscos y a icnofósiles tuvo su entrada en el Museo mediante una donación y varias recolecciones llevadas a cabo a finales del siglo XIX y principios del XX. Entre estas últimas, destacan las llevadas a cabo por E. Hernández-Pacheco a principios del siglo XX como Comisionado para el estudio geológico de dichas islas por la Real Sociedad Española de Historia Natural. El material ha sido revisado taxonómicamente y documentado en su origen poniendo de manifiesto su incuestionable interés histórico.

Palabras clave: Patrimonio paleontológico, invertebrados fósiles, Islas Canarias, España, moluscos, icnofósiles.

\section{INTRODUCCIÓN}

El Museo Nacional de Ciencias Naturales-CSIC (MNCN-CSIC), conserva entre sus fondos un importante patrimonio paleontológico con ejemplares procedentes de localidades clásicas españolas y extranjeras. La entrada de este material en el Museo se remonta hasta el último tercio del siglo XVIII, momento en que las colecciones del Museo constituían el núcleo del Gabinete Real (Barreiro, 1944, 1992; Montero, 1995; Montero y Diéguez, 1996; Diéguez y Montero, 1997). El Real Gabinete de Historia Natural fue fundado en 177 I por Carlos III (Calatayud, 1986, 1988; Barreiro, 1944, 1992) y ya entonces conservaba una gran cantidad de material paleontológico, aunque básicamente de fuera de nuestras fronteras (Montero, 1995; Montero y Diéguez, 1995).
Durante la primera mitad del siglo XIX, debido a la gran cantidad de avatares de todo tipo que marcan la historia española, tanto el Real Gabinete de Historia Natural como el resto de las instituciones científicas y culturales sufren un letargo, del que saldrían con fuerza en la segunda mitad de siglo (Montero y Diéguez, 1998).

En la segunda mitad del siglo XIX, las recolecciones, compras y donaciones de material paleontológico al Real Gabinete de Historia Natural se sucedieron, encauzadas en su mayor parte por el que fue primer catedrático de Paleontología de la Universidad Central, Juan Vilanova y Piera. Aunque entran en el Gabinete muchas colecciones de localidades europeas, fundamentalmente francesas, italianas y centroeuropeas, también el material español comienza a tener una buena representación. Ya en el siglo $\mathrm{XX}$, la política de incremento continúa pero se invierte la 
tendencia que tenía el origen de las colecciones, teniendo mucha más importancia la entrada de material español que de otras partes de Europa. La Guerra Civil (19361939) supuso una ruptura general dentro del Museo, lo que afectó a sus colecciones. Éstas comenzaron a recuperarse a partir de finales de los años setenta $y$, de forma definitiva, a partir de los años ochenta.

\section{DOCUMENTACIÓN HISTÓRICA DEL MATERIAL}

Los ejemplares de invertebrados de la colección paleontológica del MNCN-CSIC procedentes de las Islas Canarias corresponden a varias entradas de diferentes recolectores en épocas distintas y son, en síntesis, las derivadas de una donación realizada en la segunda mitad del siglo XIX y de tres recolectas llevadas a cabo en la primera mitad del siglo XX.

La donación del siglo pasado consta de una única muestra de mano de una roca calcárea con restos de crustáceos y gasterópodos procedente de Tenerife, único dato que consta en la etiqueta identificativa. Por el tipo de etiqueta que posee el ejemplar, nosotros asignamos su entrada al Museo en la segunda mitad del siglo XIX, etapa en la que el valenciano Juan Vilanova y Piera estaba al frente de la colección paleontológica del entonces Real Gabinete de Historia Natural (Montero, 1995).

En cuanto a las recolectas, la que contribuye con un mayor número de ejemplares fue la realizada por Eduardo Hernández Pacheco, Jefe de la Sección de Cambios del Museo Nacional de Ciencias Naturales, en un viaje en el año 1907 para estudiar la geología de Lanzarote e islas cercanas: Alegranza y Graciosa (Anónimo, 1954; Hernández Pacheco, 1907) así como para realizar un mapa geológico de estas islas a escala 1:150.000 (Anónimo, 1907; Anónimo, 1954). El material consta en su totalidad de gasterópodos, que el autor atribuye a los géneros Helix, Stenogyra y Palmatella, y dos ejemplares, que el autor describe como: "singulares concreciones huecas llamadas barrilitos en el país, que recuerdan por su forma los canutos de ciertas langostas y cuyo modo de formarse es uno de los problemas que traemos sin resolver de la excursión" . Los ejemplares en cuestión representan nidos de Hymenoptera, atribución que ya da como suposición Hernández-Pacheco en la mencionada publicación. La edad de estos ejemplares es PlioPleistocena. Los ejemplares de Isla Graciosa, atribuibles a dos taxones también recolectados en Lanzarote, tienen una pátina calcárea que los diferencia de los recogidos en esta última isla. Las etiquetas del material de Isla Graciosa llevaban como único dato informativo la localidad, y ha sido posible su adscripción a esta recolecta de Hernández-Pacheco por la inexistencia de recolectas de otros naturalistas de material con esa procedencia.

La segunda recolecta fue realizada por Ángel Cabrera y Latorre en 1910, en Tenerife, y consta de tres valvas de ostreidos.
La tercera y última recolecta de la que nos han llegado ejemplares fue realizada en Gran Canaria y, al no existir datos de recolectores ni fechas en las etiquetas, damos dos posibles orígenes:

a) Una recogida llevada a cabo por Francisco Hernández Pacheco y Carlos Vidal Box en un viaje realizado a las islas en 1934, un año después de obtener la cátedra de Geografía Física de la Universidad de Madrid el primero de ellos.

b) Una recolecta de L. Fernández Navarro entre los años 1923 y 1926. L. Fernández Navarro fue un gran conocedor de la geología canaria, habiendo realizado numerosos viajes a las islas, desde el primero en 1906 a la Isla de Hierro comisionado por la Real Sociedad Española de Historia Natural (Martín Cardoso, 1931). De 1923 a 1926, con motivo de continuar sus estudios de la geología de las islas y de preparar una de las excursiones del XIV Congreso Geológico Internacional de 1926, recorre entre otras islas la de Gran Canaria.

La presencia de una etiqueta, asignando este material a una recolecta de J. Royo Gómez en su viaje a Francia en 1925 pensionado por la Junta para Ampliación de Estudios, ha sido descartado por nosotros, ya que Royo Gómez en su viaje hacia Francia viajó antes a Valencia y Barcelona, pero no a las Islas Canarias (Memoria JAE de 1924-1925; Memoria JAE de 1925-1926; Montero, 1996). Todo el material es de gasterópodos, probablemente pleistocenos.

\section{LISTADO DE TAXA}

\section{- DONACIÓN DE EJEMPLARES EN EL ÚLTIMO TERCIO DEL SIGLO XIX}

\section{Tenerife}

Caliza con fragmentos de Balanus sp. y gasterópodos. (MNCNI- 32352)

\section{- VIAJE DE A. CABRERA LATORRE (1910) \\ Tenerife \\ Bivalvia \\ Ostrea sp.(MNCNI- 32349; 4 ejemplares)}

\section{- VIAJE DE E. HERNÁNDEZ PACHECO (1907). \\ Lanzarote. Llanura entre Soó y Tiagua.}

Gastropoda

Rumina decollata Linneo (MNCNI-11296; 21 ejemplares); (MNCNI-11297; 14 ejemplares); (MNCNI32350; 108 ejemplares); (MNCNI-32351; 24 ejemplares)

Hemicycla sarcostoma (Webb y Berthelot) (MNCNI32348; 12 ejemplares) (Fig. 1a); (MNCNI-11294; 6 ejemplares) (Fig. 1b)

Helix sp. (MNCNI-11312; 2 ejemplares)

Theba geminata (Mousson) (MNCNI-11295; 42 ejemplares en buen estado de conservación y cuatro fragmentos). Se distinguen dos grupos de ejemplares en función de su tamaño, si bien todos pertenecen a $T$. geminata (según revisión de Gittenberger et al., 1982 y Gittenberger y Ripken, 1987) debido a la falta de quilla, que sólo aparece en una forma juvenil. La población 1 (Fig. 1d), compuesta por 13 ejemplares completos de 


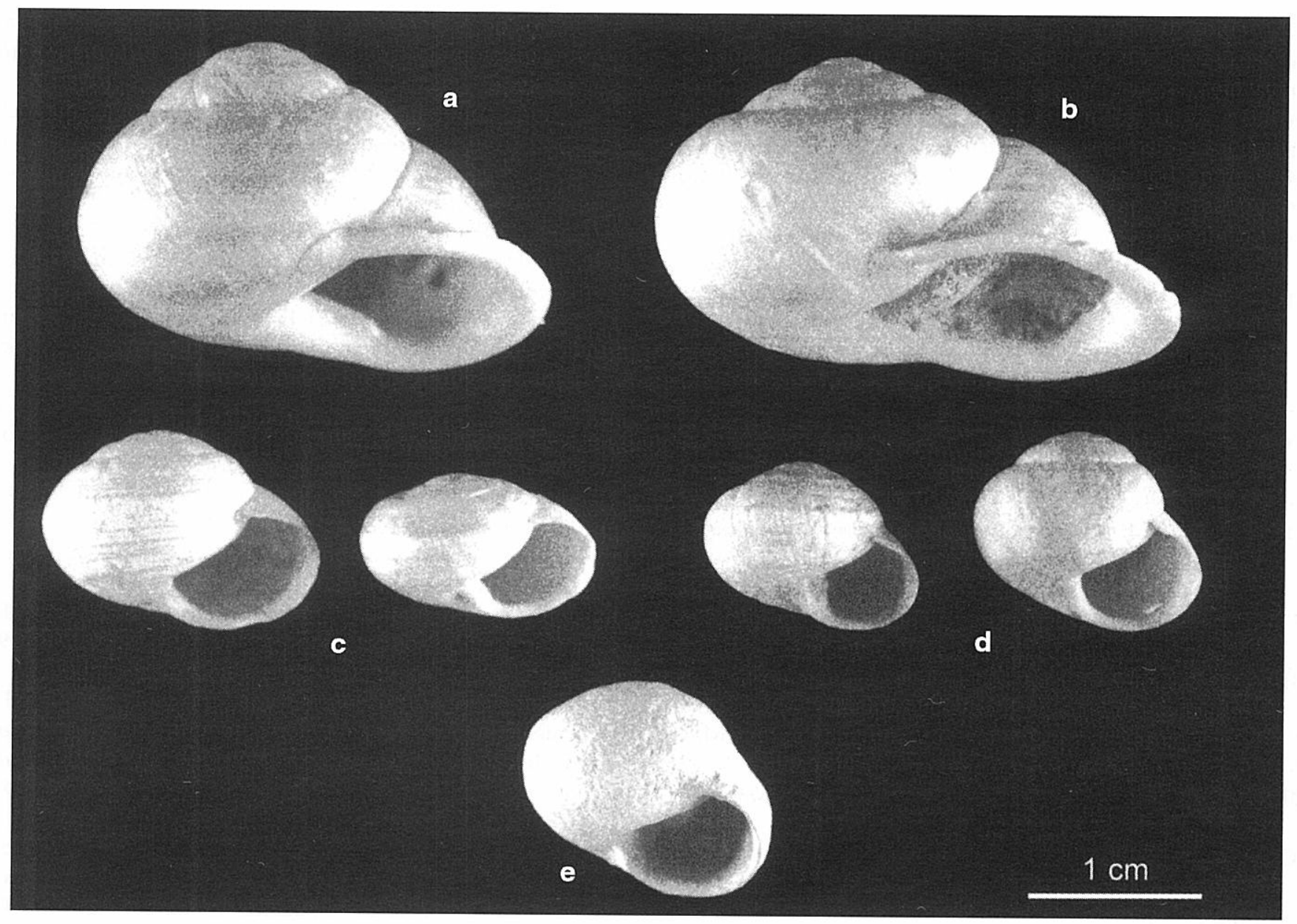

Figura 1. a. Hemicycla sarcostoma (Webb y Berthelot) (MNCNI-32348). Lanzarote. b. Hemicycla sarcostoma (MNCNI11294)). Lanzarote. c. Theba geminata (Mousson) (MNCNI- 11295). Dos ejemplares completos representativos de la población 2. Lanzarote. d. Theba geminata. (MNCNI- 11295). Forma juvenil (izquierda) y adulta (derecha) de la población 1. Lanzarote. e. Theba geminata. (MNCNI- 4830). Isla Graciosa.

a. Hemicycla sarcostoma (Webb and Berthelot) (MNCNI-32348). Lanzarote. b. Hemicycla sarcostoma (MNCNI11294). Lanzarote. c. Theba geminata (Mousson) (MNCNI- 11295). Two complete specimens representatives of the population 2. Lanzarote. d. Theba geminata. (MNCNI- 11295). Yonng (left) and adult (right) forms representatives of population 1. Lanzarote. e. Theba geminata. (MNCNI- 4830). Isla Graciosa.

formas adultas (altura máxima: 13,6 cm; mínima: 10,4 cm; media: 11,8 cm; anchura máxima: 17,8 cm; mínima:14,1 cm; media: 15,9) y un ejemplar de una forma juvenil (altura: 9,7; anchura: 13,4 cm). Además, forman parte del lote cuatro fragmentos de diversos tamaños. La población 2 (Fig. 1c) está compuesta por 28 ejemplares completos de fosmas adultas (altura máxima: 11,7 cm; mínima: 7,8 cm; media: 9,5; anchura máxima: 14,7; mínima: 10,7; media: 12,2)

Testacella sp. (MNCNI-11298; 298 ejemplares) Icnofósiles

Nidos de Hymenoptera (véspidos) (MNCNI-33133; 2 ejemplares)

\section{Isla Graciosa}

Gastropoda

Rumina decollata Linneo (MNCNI-4824; 3 ejemplares)

Theba geminata (Mousson) (MNCNI-4830) (Fig. 1e). Seis ejemplares completos, en buen estado de conservación (altura máxima: 12,2 cm; mínima: 10,7cm; media: 11,5 cm; anchura máxima: $15,5 \mathrm{~cm}$; mínima: 14,4 $\mathrm{cm}$; media: $15,1 \mathrm{~cm}$ )

- PROBABLE DONACIÓN DE L. FERNÁNDEZ NAVARRO (1923-26) O DE F. HERNÁNDEZ PACHECO Y C. VIDAL BOX (1934).

Gran Canaria. Las Palmas.

Gastropoda

Ancylus fluviatilis Müller (MNCNI- 11559; 6 ejemplares y 1 fragmento )

Lymnaea boulleti Michaud (MNCNI-1105; 2 ejemplares)

Hemicycla glasiana Shuttlew (MNCNI-29023; 1 ejemplar)

\section{CONCLUSIONES}

Los ejemplares de invertebrados fósiles canarios presentes en los actuales fondos del Museo Nacional de 
Ciencias Naturales (CSIC), aunque escasos tanto en número de ejemplares como en diversidad, tienen un interés de carácter histórico incuestionable, al haber sido resultado de recolectas de eminentes naturalistas en momentos en los que las islas estaban empezando a ser estudiadas sistemáticamente desde un punto de vista geológico. También es indudable que, dada la escasa presencia en las colecciones del MNCN-CSIC de ejemplares fósiles canarios, cualquier muestra más o menos representativa del área en cuestión tiene interés.

\section{BIBLIOGRAFÍA}

Anónimo. 1907. Comisión del Noroeste de Africa. Boletín de la Real Sociedad Española de Historia Natural, 7, 267.

Anónimo. 1954. Sucinta biografía del profesor Eduardo Hernández-Pacheco. Boletín de la Real Sociedad Española de Historia Natural, tomo extr. 80 Aniversario del nacimiento del profesor HernándezPacheco, 7-34.

Barreiro, A. 1944. El Museo Nacional de Ciencias Naturales. Consejo Superior de Investigaciones Científicas. Instituto de Ciencias Naturales "José de Acosta", Madrid, $381 \mathrm{pp}$.

Barreiro, A. 1992. El Museo Nacional de Ciencias Naturales (1771-1935). Ed. Doce Calles, Madrid, 509 pp.

Calatayud, M. A. 1986. Antecedentes y creación del Real Gabinete de Historia Natural de Madrid. Arbor, 123 (482), 9-31.

Calatayud, M. A. 1988. Pedro Franco Dávila y el Real Gabinete de Historia Natural. C.S.I.C. Museo Nacional de Ciencias Naturales, Madrid, VIII + 251 pp.

Diéguez, C. y Montero, C. 1997. La colección de invertebrados fósiles del Museo Nacional de Ciencias Naturales (CSIC). Graellsia, 53, 31-35.
Gittenberger, E. and Ripken, T. E. J. 1987. The genus Theba (Mollusca, Gastropoda, Helicidae) systematics and distribution. Zoologische Verhandelingen, 241, 1-59.

Gittenberger, E., Ripken, T. E. J. and Bueno, M. L. 1982. The forgotten Theba species (Gastropoda, Pulmonata, Helicidae). Proceedings IX International Malacological Congress (Edinburg, 1992), 145-151.

Hernández Pacheco, E. 1907. Exploración geológica de Lanzarote y de las isletas canarias. Boletín de la Real Sociedad Española de Historia Natural, 7, 339-348.

Martín Cardoso, G. 1931. El Profesor Fernández Navarro (1869-1930). Reseñas Científicas. Boletín de la Real Sociedad Española de Historia Natural, 6, 5-22.

Memoria correspondiente a los cursos 1924-1925 (1926) y 1925-1926 (1927). Junta para la Ampliación de Estudios e Investigaciones Científicas, Madrid, 480 pp.

Montero, A. 1995. La colección de invertebrados fósiles del Museo Nacional de Ciencias Naturales-CSIC. Su desarrollo histórico y museológico. Tesis Doctoral. Universidad Complutense de Madrid. 807 pp. (Inédita).

Montero, A. 1996. J. Royo y Gómez y sus viajes pensionado por la Junta de Ampliación de Estudios. Geogaceta, 19, 183-184.

Montero, A. y Diéguez, C. 1995. El interés por la Paleontología en el Siglo XVIII: Análisis del Catálogo de la Colección Franco Dávila. Boletín de la Real Sociedad Española de Historia Natural (Sección Geológica), 90, 87-94.

Montero, A. y Diéguez, C. 1996. Las ideas sobre el origen de los fósiles de Fernando López de Cárdenas, colector del Real Gabinete en la segunda mitad del siglo XVIII. Boletín de la Real Sociedad Española de Historia Natural, Tomo extr. 125 Aniversario, 518-521.

Montero, A. y Diéguez, C. 1998. La Paleontología en el Real Gabinete de Historia Natural en los siglos XVIII y XIX (1771-1895). Boletín de la Real Sociedad Española de Historia Natural (Sección Geológica), 94, 139-148. 